\section{Protein kinases mix it up}

Phenotypic diversity is the substrate for natural selection, so identifying the components that contribute to this diversity is crucial for understanding evolution. Much attention has focused on differences in gene expression between species, but to what extent do other cellular components provide phenotypic diversity? Combining the power of high-throughput proteomics and genetic screens, this paper quantifies the evolutionary potential of one such component - protein phosphorylation.

Protein phosphorylation a ubiquitous and reversible posttranslational modification that is vital for the regulation of essential cellular processes - is controlled by transient interactions between protein substrate sites and protein kinases. To examine how kinasesubstrate interactions evolve, Beltrao and colleagues used highthroughput mass spectrometry to analyse the phosphoproteomes of 3 yeast species that are separated by 400-600 million years of evolution: Saccharomyces cerevisiae, Candida albicans and Schizosaccharomyces pombe. By comparing these phosphoproteomes, these authors estimate that kinase-substrate interactions change at a rate of $\sim 1 \times 10^{-6}-1 \times 10^{-5}$ per protein per million years; the rate of change is likely to be faster if changes of phosphorylation site in the same protein are considered.
They estimated that at most, kinasesubstrate interactions change at a rate that is two orders of magnitude slower than transcription factorpromoter interactions; however, the rates of change could potentially be equal.

The authors then used data from high-throughput genetic interaction screens - based on the E-MAP strategy, which uses pairwise genetic crosses of panels of mutants to generate interaction profiles to assess whether protein kinases evolve new functions more rapidly than average genes. They found that $\sim 14 \%$ of a set of over 5,000 pairs of genes that genetically interact in $S$. cerevisiae also interact in S. pombe, but only $\sim 8 \%$ of genetic interactions that involve protein kinases were conserved. This genetic evidence suggests that protein kinases have a higher than average rate of functional divergence. The authors propose that this, together with the proteomic data, shows kinase-substrate interactions are likely to constitute an important source of phenotypic variation.
Interestingly, the authors found that although individual kinasesubstrate interactions change quickly, the overall phosphorylation levels associated with specific complexes or biological processes, such as cytokinesis or budding, are highly conserved. This observation might be consistent with a view of evolution in which modules or complexes are conserved but regulatory interactions change.

This paper provides the first quantitative estimate of the contribution of a regulatory process that does not affect gene expression to diversification. Furthermore, it shows the potential of combining physical and genetic interaction data from high-throughput analyses. In addition to examining the evolutionary potential of protein phosphorylation in more detail, future studies might use a similar approach to study the evolution of protein networks.

Mary Muers

ORIGINAL RESEARCH PAPER Beltrao, P. et al. Evolution of phosphoregulation: comparison of phosphorylation patterns across yeast species. PLoS Biol. 7, e1000134 (2009)

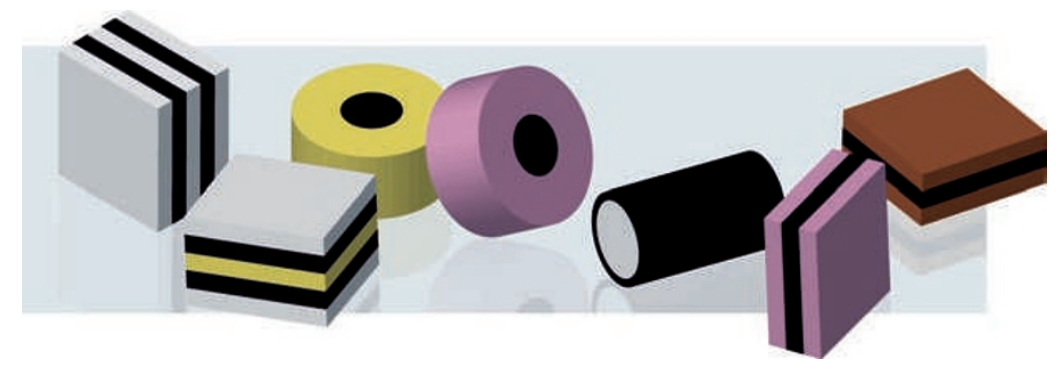

